CASE REPORT

\title{
Congenital nodular multiple glomangioma: a case report
}

\section{J S Chatteriee, A H K Youssef, R M Brown, H Nishikawa}

J Clin Pathol 2005;58:102-103. doi: 10.1136/icp.2003.014324

A 13 year old girl presented with recurrent painful "varicosities" on her right calf. These lesions were subsequently clinically diagnosed as "cavernous haemangiomas" after normal duplex scanning and were excised. Histological examination revealed multiple glomangiomas (glomus tumours). A literature review revealed only two reported cases of nodular multiple glomangioma, so that this is the third case to be reported in the literature.

\section{G} lomus tumours are benign tumours of the perivasculature. They arise from modified smooth muscle cells, called glomus cells, located in the walls of the Sucquet-Hoyer canal, a specialised arteriovenous anastomosis central to thermoregulation. ${ }^{1}$ Most tumours are less than $1 \mathrm{~cm}$ in diameter, occurring in the dermis or subcutis in the upper and lower extremities. ${ }^{1}$ There are two forms of glomus tumour, with the more common solitary variant accounting for most of the cases $(90 \%)$, and a rarer multiple variant accounting for $10 \%$ of cases ${ }^{2}$; this second form is seen most often in children and is thought to be inherited in an autosomal dominant fashion. ${ }^{1}$ The multiple variant is subdivided clinically into nodular and plaque-like lesions. Histologically, glomus tumour is composed of varying proportions of glomus cells, blood vessels, and smooth muscle. ${ }^{1}$ Depending on the predominant component, there are three variants of glomus tumour, namely: (1) angiomatoid (glomangioma) with predominant blood vessels; (2) solid (predominantly glomus cells); and (3) glomangiomyoma (predominantly smooth muscle). Multiple glomus tumours generally correspond to glomangioma.

\section{"A literature review revealed only two reported cases of this rare variant"}

In 2001, Carvalho et al reported a congenital plaque-like variant and reviewed 14 other cases. Of these 15 cases, 13 were described as the plaque-like type and two were nodular. ${ }^{2}$ In 2001, an additional case of congenital plaquelike multiple glomus tumour was reported by Lin et al. ${ }^{3}$

We report a case of congenital multiple glomangioma in a 13 year old girl, with the nodular variant. A literature review revealed only two reported cases of this rare variant.

\section{CASE REPORT}

A 13 year old girl was seen in the paediatric plastic surgery outpatient clinic in Birmingham Children's Hospital, UK, after being referred for painful varicosities on her right calf, recurring after three previous phlebectomies (histological examination was not performed). These bluish "venous" lesions had been present since birth. The patient was experiencing pain at the site of the lesions. On standing the lesions would dilate. After each excision the lesions reappeared. The patient's mother, grandmother, great grandmother, and great aunt each had similar lesions at different anatomical locations. Although there was no histological diagnosis for these lesions, the clinical picture supports an autosomal dominant inheritance.

On clinical examination, there appeared to be varicosities along the long saphenous axis in her right calf (fig 1). Duplex scanning showed normal superficial and deep veins in the right leg. At this point it was assumed that the lesions were in fact scattered cavernous haemangiomata. Excision of the lesions was carried out by the vascular surgery department. At surgery, there appeared to be multiple subcutaneous haemangiomata, "like isolated knotted varicose veins", which were excised with skin ellipses and subcutaneous tissue from the right upper part of the thigh, popliteal fossa, and medial calf. Eight lesions were excised in total.

\section{PATHOLOGICAL FINDINGS}

Macroscopic examination showed 10 pieces of fibro-fatty tissue, the largest of which measured $2.5 \times 2.5 \times 2 \mathrm{~cm}$. The smallest measured $1.3 \times 0.7 \times 0.4 \mathrm{~cm}$. Most of them showed bluish vessels. Microscopy revealed multiple diffuse lesions, present both within the dermis and deep within the subcutaneous tissue fragments, and unlikely to be excised completely. These lesions were formed of dilated vessels, with a surrounding collar of regular round cells with round nuclei

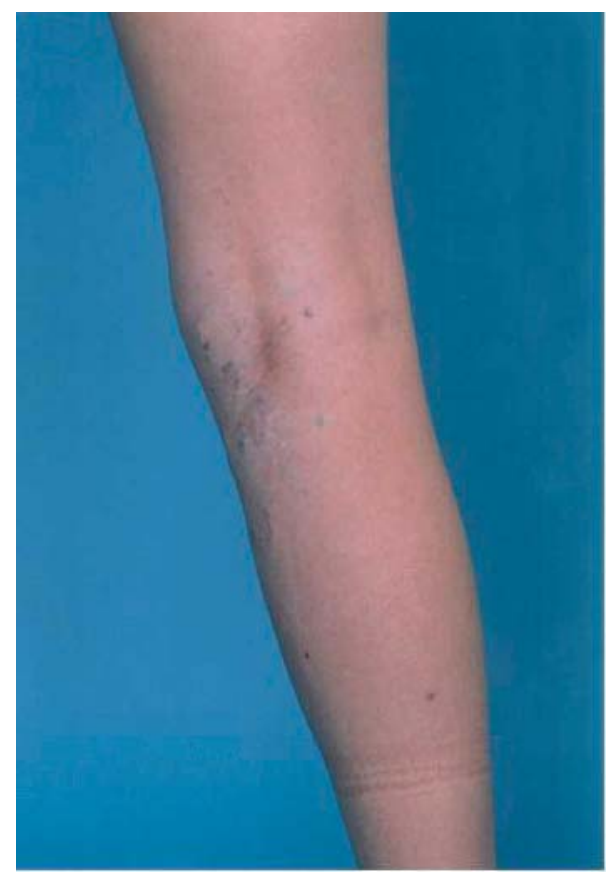

Figure 1 The patient's right calf, showing varicosities along the long saphenous axis. This photograph is reproduced with the full consent of the patient. 


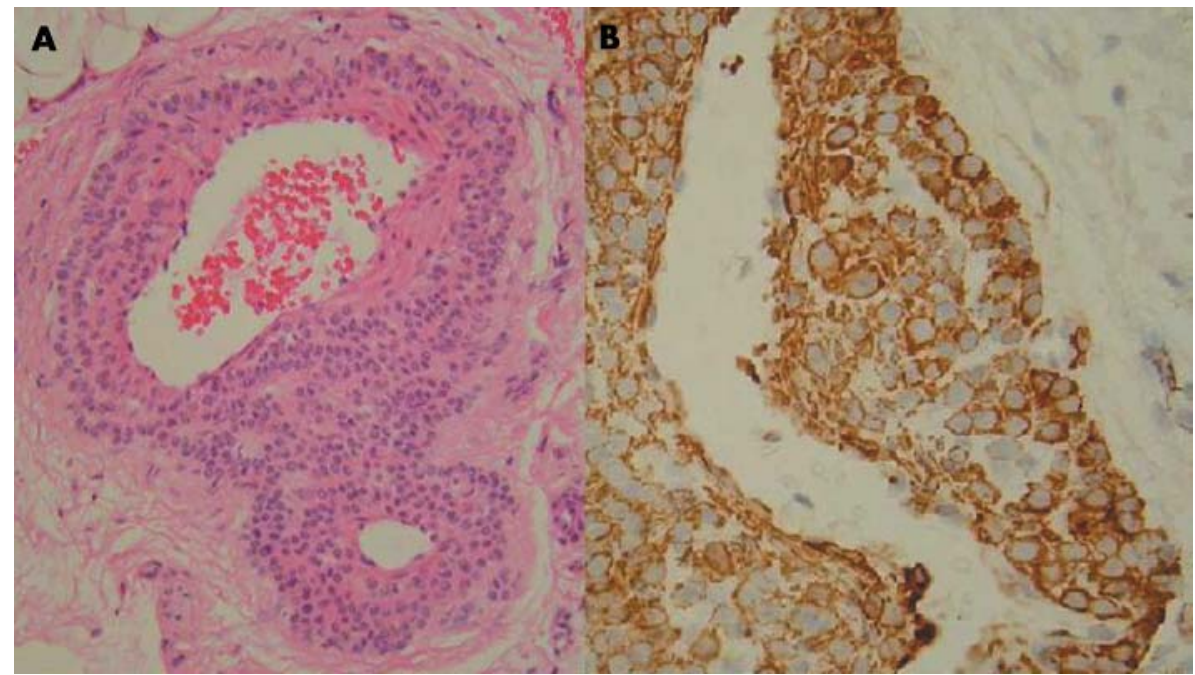

Figure 2 (A) Low power view of a lesion formed from dilated vessels, with a surrounding collar of regular round cells with round nuclei and eosinophilic cytoplasm (haematoxylin and eosin stain). (B) The tumour cells were positive for smooth muscle actin (immunohistochemical stain).

and eosinophilic cytoplasm (fig 2A). There was no mitotic activity or atypia. These features were consistent with the angiomatoid variant of glomus tumour (glomangioma). The presence of the tumour within the subcutaneous tissue is in keeping with what has been described as "infiltrating glomus tumour", which implies difficulty in complete excision and the possibility of recurrence.

The tumour cells were immunoreactive for smooth muscle actin (fig 2B). The periodic acid Schiff stain distinctively highlighted the cytoplasmic membrane of the tumour cells. These immunohistochemical and special stains added support to the diagnosis.

\section{DISCUSSION}

Glomangioma has an early onset, with one third of cases presenting before 20 years of age. ${ }^{4}$ Familial cases have been reported with autosomal dominant transmission, incomplete penetrance, and variable expression. In 2000, Pena-Penabad et al reported two cases of familial multiple glomangiomas, but the lesions were not present from birth in the first patient and the second patient presented for consultation as an elderly adult. ${ }^{5}$ There have been several papers describing glomangiomas in the knee region, ${ }^{6-9}$ but all four papers describe a solitary tumour, which is different from the multiple type reported here. Clinically, glomangiomas appear as red to blue compressible papulonodules. ${ }^{2}$ Although solitary glomus tumours often produce pain, multiple glomangioma usually does not. In our case, the patient presented with pain. In most cases there are fewer than 10 lesions. Of the 15 cases of congenital glomangioma presenting in childhood found in the literature, 13 were described as the plaque type and two as nodular. Our patient presented in childhood with painful, bluish nodules in her right lower extremity, fitting in with the nodular multiple variant.

This is the third case in the literature of congenital multiple glomangioma, nodular type, presenting in childhood. The presence of the tumour within the subcutaneous tissue explains why it recurred.

\section{Take home messages}

- We describe a rare case of congenital multiple glomangioma, nodular type, presenting in childhood-only the third to be reported

- The fumour was present within the subcutaneous tissue, causing it to recur

\section{Authors' affiliations}

J S Chatterjee, A H K Youssef, R M Brown, H Nishikawa, Department of Plastic Surgery and Pathology, Birmingham Children's Hospital, Birmingham, UK

The patient gave full consent for the reproduction of the photograph.

Correspondence to: Mr J S Chatterjee, 42 Badger Park, Broxburn, Edinburgh, West Lothian EH52 5GZ, UK; justinchatterjee@aol.com

Accepted for publication 18 June 2004

\section{REFERENCES}

1 Fletcher C. Tumours of blood vessels and lymphatics. In: Diagnostic histopathology of tumors. Edinburgh: Churchill Livingstone, 2000:75-6.

2 Carvalho VO, Taniguchi K, Giraldi S, et al. Congenital plaquelike glomus tumor in a child. PediatrDermatol 2001;18:223-6.

3 Lin T-M, Tsai C-C, Tsai K-B, et al. Congenital multiple plaque-like glomangiomyoma in trunk-a case report. Kao Hsiung I Hsueh Ko Hsueh Tsa Chih 2001;17:377-80.

4 Goodman T, Abele D. Multiple glomus tumours. Arch Dermatol 1971;103:11-22.

5 Pena-Penabad C, Garcia-Silva J, del Pozo J, et al. Two cases of segmental multiple glomangiomas in a family: type 1 or type 2 segmental manifestation? Dermatology 2000;201:65-7.

6 Hardy P, Muller PG, Got C, et al. Glomus tumor of the fat pad. Arthroscopy 1998;14:325-8.

7 Negri G, Schulte M, Mohr W. Glomus tumor with diffuse infiltration of the quadriceps muscle: a case report. Hum Pathol 1997;28:750-2.

8 Mabit C, Pecout C, Arnaud JP. Glomus tumor in the patellar ligament: a case report. J Bone Joint Surg Am 1995;77:140-1.

9 Oztekin H. Popliteal glomangioma mimicking Baker's cyst in a 9-year-old child: an unusual location of a glomus tumor. Arthroscopy 2003;19:1-5. 
3 Costes V Duchayne E, Taib J, et al. Intrasinusoidal bone marrow infiltration: common growth pattern for different lymphom subtypes. Br J Haematol 2002;119:916-22.

4 Kent SA, Variakojis D, Peterson LC. Comparative study of marginal zone lymphoma involving bone marrow. Am J Clin Pathol 2002;117:698-708.

5 Audouin J, Le Tourneau A, Molina T, et al. Patterns of bone marrow involvement in 58 patients presenting primary splenic marginal zone ymphoma with or without circulating villous lymphocytes. Br J Haematol 2003;122:404-12.

\section{BOOK REVIEW}

\section{Molecular approaches to malaria}

Edited by I W Sherman. Washington DC: ASM Press, 2005, pp 542. ISBN 1555813305

In a world where most up-to-date information can be gained easily and cheaply by a PubMed search for the most appropriate review article on any subject, the role of the textbook increasingly appears redundant. Books such as Molecular approaches to malaria, however, reaffirm the value of a good textbook.

Malaria continues to be one of the most important causes of death and illness worldwide. There are about half a billion cases of malaria and around two million deaths occur annually, mostly in children in sub-Saharan Africa. Despite having effective treatment for malaria (quinine) for over 300 years and a knowledge of the biology of the causative agent for over a hundred years, the number of deaths from malaria continue to rise. Large investments have been made in malaria research during the past two decades Consequently, the disciplines of cell and molecular biology of Plasmodium falciparum have expanded dramatically, with, for example, the sequencing of the $P$ falciparum genome, novel insights into metabolism and also the molecular basis of drug resistance.
The recent development of fosmidomycin, a new malaria drug, gives an excellent example of how understanding the apparently obscure topic of apicoplast metabolism can lead to the identification of a new class of drugs that are now in clinical development. This new knowledge of the biology of malaria along with rapidly expanding knowledge of gene expression and proteomics will continue to provide valuable insights into the pathogenesis of malaria and further treatments for this disease. Molecular approaches to malaria brings together all these topics in one clear and comprehensive textbook.

There is also the fascination of the biology of an organism with many stages, which is perfectly adapted to live in both vertebrate and invertebrate hosts in different stages of its complex life cycle. This book is not intended for a casual non-specialised readership, but should prove invaluable to anyone working in the field of malaria or with a special interest in this intriguing and important disease.

This book is structured into six sections with 27 chapters. These chapters cover a broad range of topics from cell biology to metabolism and immune evasion. There are also useful chapters on the use of internet resources for malaria research and techniques for genetic manipulation of $P$ falciparum.

As with any multi-authored textbook, the chapters are of variable quality, but on the whole the writing is first class, albeit dense The chapters are written by the appropriate world experts, complete with up-to-date references (many from 2005). The editing is excellent, with little overlap between chapters, coherent style and clear indexing. My only (small) gripe is that all the colour plates are in one section, which means switching pages often. This is a comprehensive text book that is authoritative and easy to read, and I look forward to putting my copy to good use in the future.

T Planche

\section{CALENDAR OF EVENTS}

\section{Combined adult and congenital cardiovascular pathology course}

29 November-1 December 2006, Royal Brompton \& Harefield NHS Trust, London, UK.

This "hands-on" course approaches in detail the problems that face the diagnostic pathologist when dealing with cardiovascular pathology, both congenital and adult. The approach to a cardiac autopsy and sudden death will be emphasised. Cardiac specimens will be made available for analysis and there will also be video demonstrations. A slide seminar with slides is included. The course is aimed at trainees studying for the MRC PATH, and also at senior pathologists who wish to update their knowledge. The course fee is $£ 400$ (including coffee, tea and lunch). There is a special fee of $£ 300$ for junior doctors in training.

Further details: Short Course Office, National Heart and Lung Institute, Dovehouse Street, London SW3 6LY, UK; Tel: +44 (0)20 7351 8172; Fax: +44 (0)20 7351 8246; Email: shortcourse.nhli@ic.ac.uk

\section{CORRECTION}

doi: 10.1136/jcp.2004.019323.corrl

There was an error in reference 9 of the article by Chatterjee JS, Youssef AHK, Brown RM, et al. Congenital nodular multiple glomangioma: a case report. $J$ Clin Pathol 2005;58:102-10. The correct reference should be as follows: Öztekin HH. Popliteal glomangioma mimicking Baker's cyst in a 9-year-old child: an unusual location of a glomus tumor. Arthroscopy 2003;19:e67-e71. 


\section{PostScript}

\section{LETTER TO THE EDITOR}

\section{An uncommon pattern of cardiac invasion in hepatocellular carcinomaHCC, hepatocellular carcinoma}

Hepatocellular carcinoma (HCC), the most common primary malignant tumour of the liver, is a highly vascular neoplasm usually arising in a cirrhotic liver. In addition to the diagnosis of primary lesion, an assessment for extrahepatic metastasis is needed. Herein, an extremely uncommon pattern of heart invasion in a patient with advanced HCC is described.

A 75-year-old man was admitted to the Hospital of Zumarraga, Zumarraga, Spain, for moderate abdominal pain, asthenia and weight loss. He denied having dyspnoea, orthopnoea, cough, palpitation or peripheral oedemas. Three years earlier, HCC had been diagnosed in the eighth segment, and the patient had received several courses of transcatheter arterial chemoembolisation. An objective examination showed hepatosplenomegaly and ascites, with no signs of cardiac failure. Temperature, blood pressure and cardiac frequency were normal. Laboratory data showed raised serum levels of aspartate transaminase and afetoprotein. Electrocardiogram and chest $x$ ray showed no abnormalities. A total-body computed tomography scan highlighted diffuse infiltration of hepatic lobes (fig l), mesenteric nodules with ascites (suggestive of carcinomatosis), multiple lymphadenopathy in celiac trunk and portocava veins and a mass in anterior pericardium (fig 2) with no pericardial or

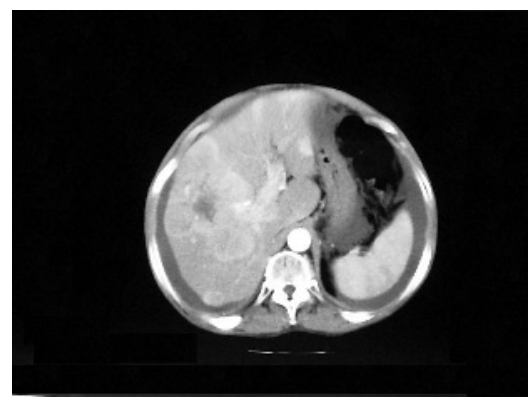

Figure 1 Computed tomography scan of the abdomen showing diffuse infiltration of both hepatic lobes.

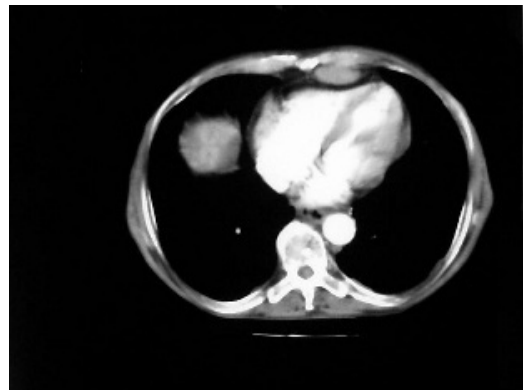

Figure 2 Computed tomography scan of the chest showing a rounded, oval-shaped mass in the anterior pericardium.

pleural effusion. Peritoneal fluid showed an exudate but no malignant cells. The cava and suprahepatic veins, and right atrium did not show evidence of tumoral invasion. The patient died 2 weeks later from acute renal failure. An autopsy was not authorised.

Practically all malignant secondary neoplasms may affect the heart. Metastatic spreading to the heart has been identified in about one fifth of all patients who have metastatic cancer. The pericardium may be invaded, mainly by carcinoma of the lung followed by breast carcinoma, lymphoma and leukaemia. Rare metastatic neoplasms of the pericardium include carcinomas of the digestive tract, such as colon, oesophagus, stomach and others. ${ }^{1}$ HCC spreads most often to the lungs, peritoneum, adrenal glands and bones. The incidence of cardiac metastasis of HCC has been reported in up to $4.1 \%$ of patients. ${ }^{2}$ Classically, several routes of pericardiac involvement in malignancy have been described, ${ }^{1}$ such as haematogenous route, by direct extension from intrathoracic cancer, by retrograde lymphatic embolisation from the mediastinal lymph nodes, by continuous spreading through the diaphragm with infiltration of the left atrium and by tumour thrombus in the venous system. The mechanism of cardiac involvement in HCC is related to the marked propensity of this tumour for vascular invasion and extension, such as tumour thrombus including the hepatic vein and the inferior vena cava, thus easily reaching the right cardiac cavities. ${ }^{2}{ }^{3}$ Pericardiac involvement through direct invasion of the anterior mediastinum is another rare mechanism that has been reported in a few patients with advanced HCC after several courses of transcatheter arterial chemoembolisation. ${ }^{4}$ This patient represents an extremely unusual pattern of cardiac involvement from HCC because of intravascular tumour thrombus or growth; transdiaphragmatic infiltration or direct extension from mediastinum or lymph nodes was absent. Thus, the route of isolated pericardial metastasis was probably haematogenous.

E Anton

Department of Internal Medicine, Hospital of Zumarraga, Zumarraga, Spain

A Astiazaran

Department of Radiology, CHDonostia, Donostia, Spain

Correspondence to: $E$ Anton, Department of Internal Medicine, $B^{\circ}$ Argixao s/n, 20700-Zumarraga (Guipuzcoa), Spain; ejaaranda@hotmail.com doi: 10.1136/jcp. 2005.035238

Accepted for publication 22 November 2005

Competing interests: None declared.

\section{References}

1 Hanfling SM. Metastatic cancer to the heart: review of the literature and report of 127 cases. Circulation 1960;22:474-83.

2 Kojiro M, Nakahara H, Sugihara S, et al. Hepatocellular carcinoma with intra-atrial tumor growth. A clinipathologic study of 18 autopsy cases. Arch Pathol Lab Med 1984;108:989-92.

3 Nakashima T, Okuda K, Kojiro M, et al. Pathology of hepatocellular carcinoma in Japan: 232 consecutive cases autopsied in ten years. Cancer 1983:51:863-77.

4 Huang CC, Ng WW, Chiang JH, et al Hepatocellular carcinoma with mediastinal and pericardial invasion: report of two cases. Chin Med J 1999:62:891-5.

\section{CORRECTION}

doi: 10.1136/jcp.2004.019323.corr2

We regret that there was an error in the correction that was published in the last issue. Reference 9 in the article by Chatterjee JS, Youssef AHK, Brown RM, et al. Congenital nodular multiple glomangioma: a case report. $J$ Clin Pathol 2005;58:102-10 should read as follows: Öztekin HH. Popliteal glomangioma mimicking Baker's cyst in a 9-year-old child: an unusual location of a glomus tumor. Arthroscopy 2003;19:e67-e71. 